\title{
Intestinal obstruction mimicking intra-abdominal hemorrhage in a teenager with familial thrombocytopenia: a case report
}

\author{
Sutaryo, MD; Kaiser Ali, MD; Untung Widodo, MD; Nunik Agustriyani, MD; \\ Edy Moeljono, MD; Mark Belletrutti, MD
}

I

nherited bleeding disorders are a heterogeneous group of disease that is complicated by hemorrhagic episodes, spontaneous, and posttraumatic. Included in this category of conditions are Familial Thrombocytopenias (FT). FT are a heterogeneous group of inherited disorders that vary in severity with regard to the degree of reduction in platelet counts and in expression of bleeding manifestations. As in acquired thrombocytopenias, FT patients are at risk for hemorrhagic complications. Rarely, patients with inherited non-thrombocytopenia bleeding disorders can develop complications of acute abdomen ${ }^{1,2}$ which present as intraabdominal hemorrhage. We report a case of intestinal obstruction mimicking an uncomplicated intraabdominal hemorrhage in a teenager with FT.

\section{The case}

A 13 year-old teenager from Saudi Arabia with a sketchy history of prior appendectomy and thrombocytopenia was referred to Intensive Care Unit, Dr. Sardjito Hospital, Yogyakarta with abdominal pain suspected of intraabdominal hemorrhage. Two days prior to the onset of abdominal pain, there was a vague history of abdominal trauma. His siblings have thrombocytopenia. Platelet count was $35 \times 10^{3} / \mathrm{ml}$. Abdominal $\mathrm{x}$-rays showed air fluid levels compatible with ileus; abdominal ultrasonogram findings were compatible with intraabdominal bleeding. He was then diagnosed as having intra-abdominal hemorrhage secondary to FT.

On physical examination in the ICU, he looked weak but still conscious, pulse rate was $120 / \mathrm{min}$, respiration rate $32 / \mathrm{min}$, temperature $38.5^{\circ} \mathrm{C}$, with a blood pressure of 100/60 mmHg. Abdomen was distended with generalised tenderness, increased percussion resonance above the level of the umbilicus, and markedly decreased bowel sounds. The hypogastric area was swollen, firm, and tender. No masses or other abnormalities were noted on rectal examination. No petechiae or bruising of the skin or mucus membranes were noted.

Investigations on admission to ICU revealed severe thrombocytopenia (platelets $4 \times 10^{3} / \mathrm{ml}$ ), mild anemia $(\mathrm{Hb} 11 \mathrm{~g} / \mathrm{dl})$, and borderline leukocytosis

From the Departments of Child Health, Division of Oncology and Hematology (S); Intensive Care (UW); Surgery (NA); Radiology(EM), Sardjito Hospital, Gadjah Mada University, Yogyakarta, Indonesia and Department of Child Health, College of Medicine, University of Saskatchewan (KA, MB).

Reprint requests to: Sutaryo, MD, Division of Oncology and Hematology, Department of Child Health, Medical School, Gadjah Mada University, Sardjito Hospital, Yogyakarta, Indonesia. Tel. 62-274-561616, Fax. 62-274-583745. 


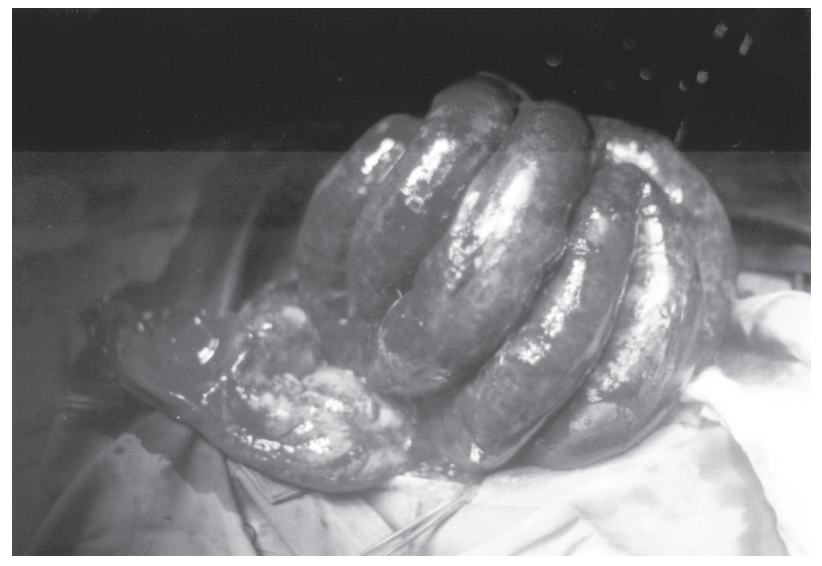

Figure 1. Several loops of ileum were necrotic

(WBC $11.4 \times 10^{3} / \mathrm{ml}$ ) with a preponderance of neutrophils. There was prolongation of bleeding time ( $>$ 15 minutes), INR (2.1) and aPTT (36.6 seconds). Abdominal X-rays showed multiple air fluid levels and loops of distended bowel with marked haustrations. Chest $\mathrm{x}$-ray, serum electrolytes, blood urea nitrogen and creatinine were within normal limits. Repeated abdominal ultrasonogram showed minimal amounts of intraperitoneal and retroperitoneal fluid, distended small bowel, and dilated pelvis of the right kidney.

In viewing his family history, the patient was considered to have FT with an intraabdominal hemorrhage. However, based on his lower abdominal findings, fever, neutrophilia, and coagulopathy, intestinal obstruction due to previous abdominal surgery was also suspected.

Oral intake was withheld, nasogastric tube and urinary catheters were inserted, oxygen was administered at 5 Litres/min via nasal prongs, and he was started on i.v. ringer's lactate to correct hypovolemia as well as for maintenance of hydration. To treat possible sepsis, he was given the following i.v. antibiotics: cefotaxim $1 \mathrm{mg} / 24 \mathrm{hrs}$ and metronidazole $500 \mathrm{mg}$ every 8 hours. On the hospital day, cefotaxime was replaced by Claforan $1 \mathrm{mg}$ every 8 hours, and and amikacin $300 \mathrm{mg}$ every 12 hours was added. Supportive medications included Novalgin $500 \mathrm{mg}$ i.v., Gastridin (ranitidine) $50 \mathrm{mg}$ every 8 hours, vitamin C $200 \mathrm{mg}$ every 8 hours, and cimetidine 1 vial every 8 hours.

Thrombocytopenia, coagulopathy and anemia were managed with repeated transfusions of plate-

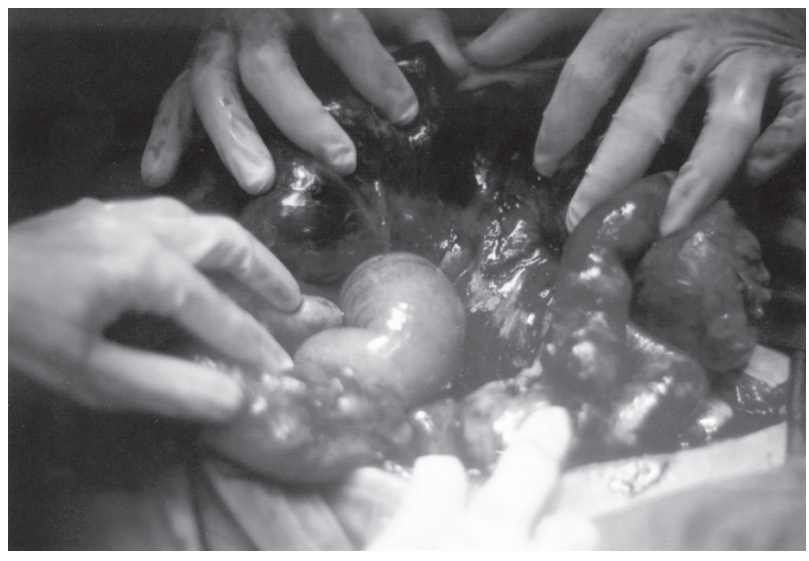

Figure 2. After releasing of the fibrous band, a segment of the bowel appeared infarcted or non viable.

lets, fresh frozen plasma, and fresh whole blood respectively. Other medications included i.v. vitamin $\mathrm{K} 10 \mathrm{mg}$ and hydrocortisone $100 \mathrm{mg}$ every 6 hours were given. Hydrocortisone was substituted by dexamethasone on the second hospital day.

On the second hospital day, he underwent a laparotomy. Following release of adhesions to the omentum, ileum and sigmoid colon, serosanguineous intraperitoneal fluid appeared which was then aspirated. Resection of a meter-long segment of infarcted bowel wrapped around a fibrous band one meter proximal to the ileo-cecal junction was performed, followed by end-to-end ileal anastomosis (Figures 1-4). The stomach, liver and spleen were normal. Approximately $1300 \mathrm{ml}$ of intraoperative blood loss was replaced by blood component transfusion.

Immediately after surgery, while on ventilatory support, his oxygen requirements increased significantly. A chest $\mathrm{x}$-ray revealed bilateral fluffy infiltrates compatible with pulmonary hemorrhage (Figure 5).

This complication was managed by increasing his $\mathrm{FiO}_{2}$ to $100 \%$ and readjusting his ventilator settings. He gradually improved, was extubated on the $6^{\text {th }}$ hospital day, and transferred to the ward in a stable condition. His clinical management is summarized in Figure 6.

\section{Discussion}

Primary thrombocytopenia is an uncommon cause of gastrointestinal (G.I.) bleeding ${ }^{3}$. In a retrospective review of 433 Danish children diagnosed with 


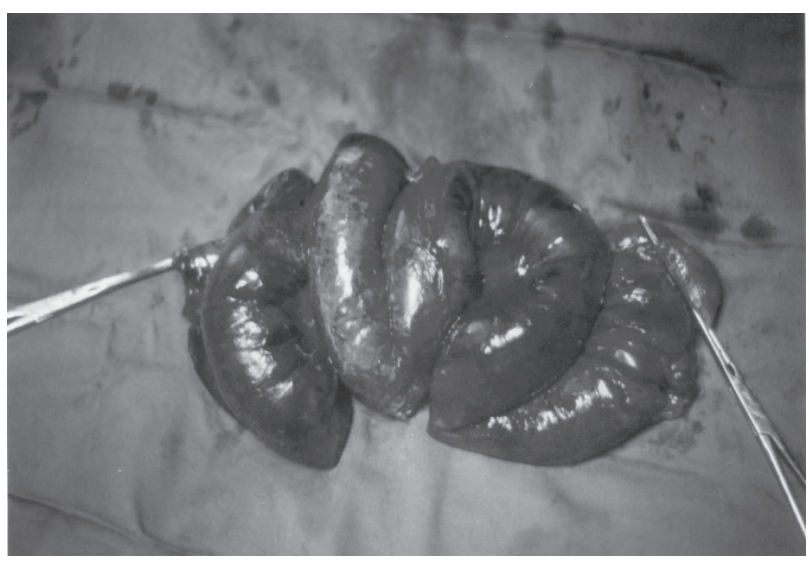

Figure 3. The segment of necrotic bowel $(100 \mathrm{~cm})$

thrombocytopenia between 1959-1969, only 22 (5\%) presented with gastrointestinal hemorrhage ${ }^{4}$. In another study of 458 consecutive patients referred for esophago-gastroduodenoscopy, only 7 (1.5\%) underwent endoscopy for G.I. hemorrhage associated with severe thrombocytopenia (mean platelet count $\left.=15 \times 10^{3} / \mu \mathrm{l}\right) .{ }^{5}$ Endoscopies performed within 24 hours of correction of thrombocytopenia in this study revealed numerous superficial upper G.I. mucosal lesions such as petechiae, ecchymoses, and erosions, but no other pathological findings.

The apparent diagnosis in our patient was that of an active intraabdominal bleeding however, it did not explain the fever, the firmness of his lower abdomen, or the coagulopathy. The differential diagnosis of these latter signs would include, in this case,such conditions as typhoid fever ${ }^{6}$ and dengue hemorrhagic fever. ${ }^{7}$ Upon arrival at DSUH, the patient was accompanied by his mother and uncle. However, they were unable to provide a complete history of his thrombocytopenia and its prevalence in the family. On admission to the ICU, it was not clear whether his thrombocytopenia was immune or non-immune of origin. A therapeutic trial of $\mathrm{ABO}$ compatible random single donor platelet transfusions on admission resulted in a rise in platelet count to $66 \times 10^{3} / \mathrm{ml}$ (Figure 6), ruling out the possibility of significant anti-platelet antibodies. Thereafter, platelet transfusions were administered from once daily to every two days in order to maintain his platelet count above a critical threshold of $50 \times 10^{3} / \mathrm{ml}$ to prevent recurrence of gastrointestinal hemorrhage. ${ }^{8}$.

With subsequent receipt of fax documents, followed by the arrival of the patient's father several days

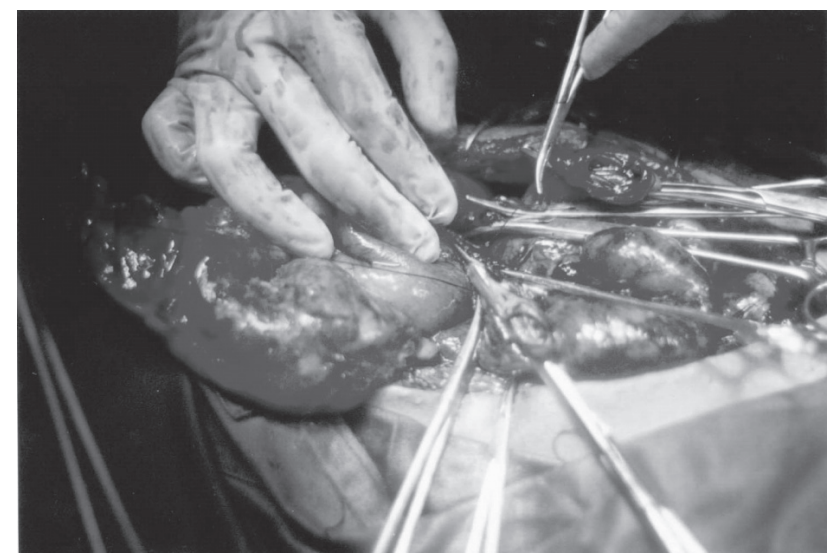

Figure 4. Remaining healthy bowel

later, details of the FT and of the patient's surgery for ruptured appendix $51 / 2$ months earlier in Riyadh, Saudi Arabia, were obtained. A discharged letter provided to the parents by a Consultant Hematologist in King Khalid Hospital, Riyadh, recommended that the patient be treated with tranexamic Acid and desmopressin for bleeding episodes. Desmopressin has been shown to be effective in patients with clotting factor deficiencies and qualitative platelet disorders such as mild or moderate Hemophilia A and von Willebrand disease, ${ }^{9}$ May-Hegglin anomaly, ${ }^{10}$ Fechtner syndrome, ${ }^{11}$ Bernard-Soulier and gray platelet syndrome, ${ }^{12}$ as well as in thrombocytic alpha-delta storage pool disease. ${ }^{13}$ Tranexamic Acid has also demonstrated hemostatic effect in dysfunctional platelet states including von Willebrand disease ${ }^{14}$ and Glanzmann's thrombasthenia. ${ }^{15}$

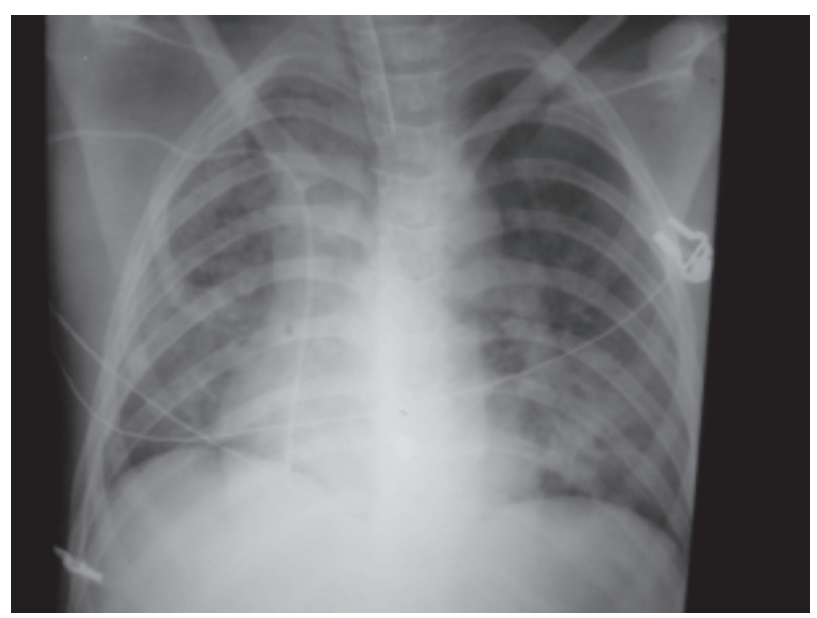

Figure 5. Chest $\mathrm{x}$-ray showing acute changes compatible with bilateral pulmonary hemorrhage 
Sutaryo et al: Intestinal obstruction and familial thrombocytopenia

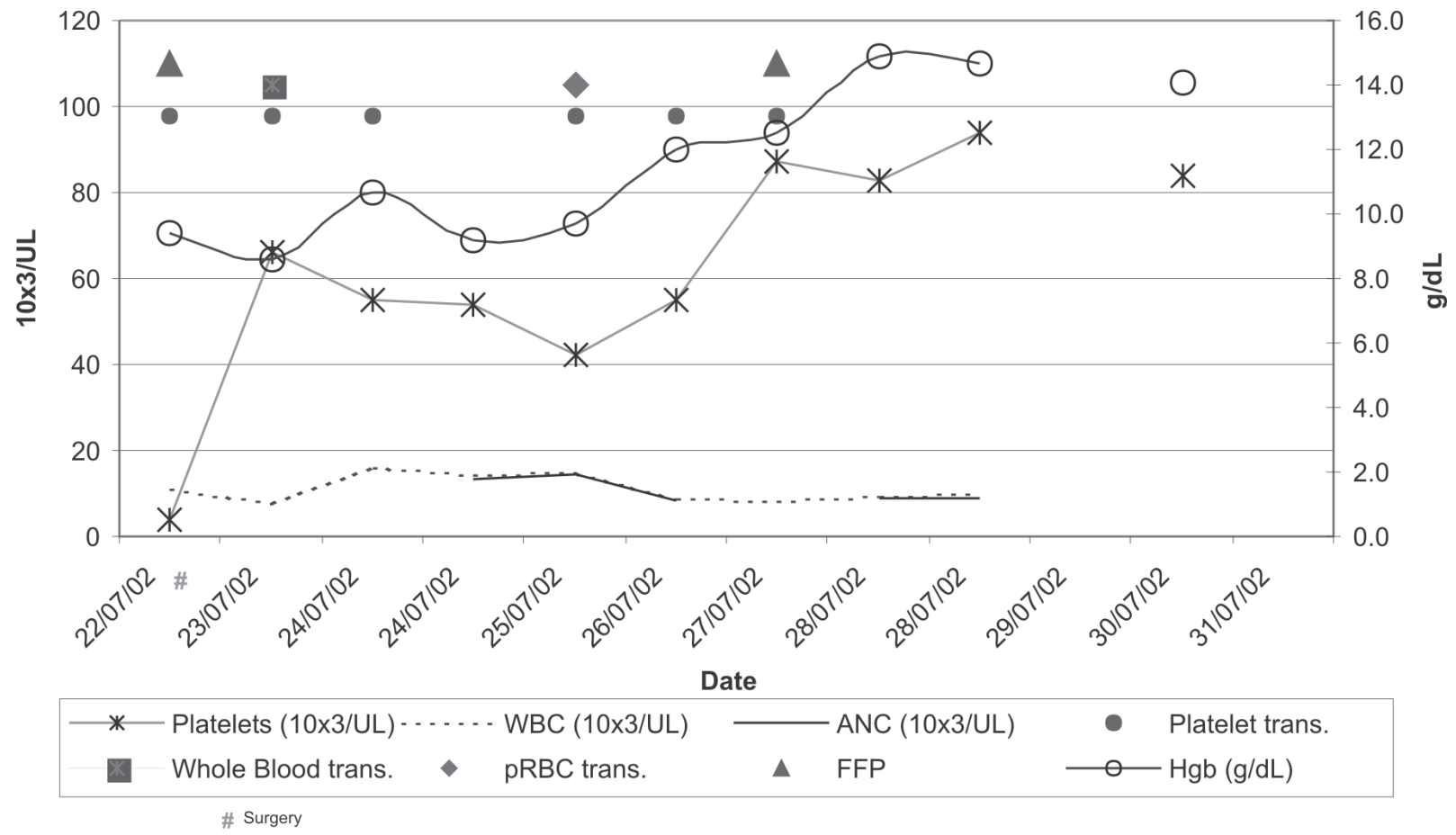

Figure 6: Graphic summary of patient's management and response to therapy

In conclusion, the practical experience gained from managing this challenging case are, first and most obvious, diagnosis to be considered in this teenager's case vis $a$ vis his acute abdomen was an intra-abdominal hemorrhage. To manage this case with only this diagnosis in mind, however, would have placed him in jeopardy since his underlying bowel obstruction unrelated to hemorrhage could potentially have ruptured, leading to a second episode, within 6 months, of perforated viscus and resultant peritonitis. Although it is rare for two concomitant diagnosis to be present in one patient, in atypical cases of acute abdomen in patients with a bleeding disorder, the possibility of a second problem underlying, or masked by, the bleeding manifestations should be kept in mind. Second, in situations where assays are not available for measurement of anti-platelet antibodies in thrombocytopenic patients, the detection of a significant rise in the platelet count one hour post-platelet transfusion is a useful method of ruling out the presence of circulating antibodies. Third, in case of limited availability of specific chemical hemostatic agents such as desmopressin or tranexamic acid for treatment of bleeding episodes in patients with non-immune thrombocy- topenia or qualitative platelet disorders, the judicious use of platelet transfusions can provide an effective therapeutic alternative.

\section{References}

1. Koussi A, Economou M, Athanasiou-Metaxa M. Intra-abdominal hemorrhage due to a ruptured corpus luteum cyst in a girl with congenital afibrinogenemia. Eur J Ped 2001;160:196.

3. Maltz GS, Siegel JS, Carson JL. Hematologic management of gastrointestinal bleeding. Gastroenterol. Clin North Am 2000;29:169-87.

4. Cohn, J. Thrombocytopenia in childhood: an evaluation of 433 patients.Scand J Hematol 1976;16:226-40.

6. Chiu CH, Tsai JR, Ou JT, Lin Ty. Typhoid fever in children: a fourteen-year experience. Acta Pediatr Taiwan 2000;41:28-32.

7. Sunarto, Sutaryo. Thrombocytopenia in dengue hemorrhagic fever. Pediatr Indones 1992;32:75-83.

8. College of American Pathologists. Practice parameter for the use of fresh-frozen plasma, cryoprecipitate and platelets. JAMA 1994;271:777-81.

9. Rodeghiero F, Castaman G, Manucci PM. Prospective multicenter study on subcutaneous concentrated 


\section{Paediatrica Indonesiana}

desmopressin for home treatment of patients with von Willebrand disease and mild or moderate hemophilia A. Thromb Haemost 1996;76:692-6.

10. Everlien M, Knoch K, Farah I, Greve H. Coronary bypass surgery in May-Hegglin anomaly. Deutsche medizinische Wochenschrift 2001;126:47-9.

12. Martinez-Murillo C, Quintana-Gonzalez S, AmbrizFernandez R, Arzate-Hernandez G, GuitierrezRomero M, Gaminio- Gomez E. Utility of desmopressin in 4 cases of thrombocytpathies associated with giant platelets. Rev Invest Clin 1997;49:281-6.
13. Kosch A, Kehrel B, Nowak-Gottl U, Haberle J, Jurgens $\mathrm{H}$. Thrombocytic alpha-delta storage pool disease: shortening of bleeding time after infusion of 1desamino-8-D-arginine vasopressin. Klin Padiatr 1999;211:198-200.

14. Stubbs M, Lloyd J. A protocol for the dental management of von Willebrand's disease, hemophilia A and hemophilia B. Aust Dent J 2001;46:37-40.

15. Rakocz M, Lavie G, Martinowitz U. Glanzmann's thrombasthenia: the use of autologous fibrin glue in tooth extractions. ASDC J Dent Child 1995;62:129-31. 